essential compared with that of the dilution with other substances. Change of the phase from solid to liquid gives a drastic change of the yield of ${ }^{115 \mathrm{~m}} \mathrm{In}^{3+}$ ion.

\section{ACKNOWLEDGMENTS}

The author's thanks are due to the kind encouragement of Dr. N. Shibata and Dr. H. Amano of the Japan Atomic Energy Research Institute. This work was supported by kind cooperation of the members of Electron Linear Accelerator of JAERI.

* Present address: Department of Chemistry, Faculty of
Science, Tohoku University, Sendai, Japan.
(a) B. Pontècorvo and A. Lazard, Compt. Rend. 208, 99
(1939); (b) G. B. Collins, B. Waldman, E. M. Stubblefield, and M. Goldhaber, Phys. Rev. 55, 507 (1939).

2 (a) G. Harbottle, Nucleonics 12, 64 (1954); (b) N. Ikeda and K. Yoshihara, Radioisotopes 7, 11 (1958); (c) K. Yoshihara, Isotopes Radiation 3, 276 (1960); (d) A. Veres, Intern. J. Appl. Radiation Isotopes 14, 123 (1963).

${ }^{3}$ J. L. Burkhardt, E. J. Winhold, and T. H. Dupree, Phys. Rev. 100,199 (1955).

${ }^{4}$ C. P. Demetroulas and P. N. Dimotakis, J. Inorg. Nucl. Chem. 28, 2756 (1966).

${ }^{5}$ K. Saito and M. Tsuchimoto, J. Inorg. Nucl. Chem. 25, 1245 (1963).

${ }^{6}$ M. L. Wiedenbeck, Phys. Rev. 68, 237 (1945); and also see W. C. Miller and B. Waldman, ibid. 75, 425 (1949).

${ }^{7}$ L. I. Schiff, Phys. Rev. 83, 252 (1951) ; see also J. D. Lawson, Nucleonics 10, 61 (1952) for correction in the case of somewhat thick target.

${ }^{8}$ This formula will be discussed in the report published later.

${ }^{9}$ K. Yoshihara and M. H. Yang, Inorg. Nucl. Chem. Letters $5,389(1969)$.

THE JOURNAL OF CHEMICAL PHYSICS

VOLUME 52 , NUMBER 6

15 M A R C H 1970

\title{
Perturbation Theory for Intermolecular Forces Including Exchange
}

\author{
H. N. W. LEKKERKERKER AND W. G. LAIDLAW \\ Department of Chemistry, University of Calgary, Calgary 44, Alberla, Canada.
}

(Received 29 August 1969)

\begin{abstract}
Generalized solutions to the Eisenschitz and London perturbation equations are derived. It is pointed out that the results obtained in the formalisms proposed by Hirschfelder (HAV), by Hirschfelder and Silbey, by Murrell and Shaw, and by Musher and Amos are special cases of the generalized treatment.
\end{abstract}

\section{INTRODUCTION}

The recent literature contains a number of proposals for including the exchange effect in perturbation formalisms for short-range intermolecular forces. ${ }^{1}$ Since most of these methods give different results for second- and higher-order energies, one might well ask which of the perturbation methods is most satisfactory. Certain et $a l{ }^{2}$ have tried to answer this question on the basis of numerical calculations. Aside from their numerical comparison one might also examine the theoretical basis of the various approaches in an attempt to elucidate their relation to one another. In this paper generalized solutions to the perturbation equations originally derived by Eisenschitz and London ${ }^{3}$ (see also Van der Avoird ${ }^{4}$ ) will be obtained and it will be shown that the results of various formalisms, which have currently received attention, can be related to these generalized solutions.

\section{GENERALIZED PERTURBATION TREATMENT}

Consider the interaction between two molecules $\mathrm{R}$ and T with $N_{\mathrm{R}}$ and $N_{\mathrm{T}}$ electrons, respectively, and let $\left\{\psi_{m}{ }^{\mathrm{R}}\right\}$ and $\left\{\psi_{n}^{\mathrm{T}}\right\}$ be the complete sets of orthonormal wavefunctions for these molecules. The product functions $\Phi_{i}=\psi_{m}{ }^{\mathrm{R}} \psi_{n}{ }^{\mathrm{T}}$ are eigenfunctions of the sum Hamiltonian $H_{0}=H_{\mathrm{R}}+H_{\mathrm{T}}$ with eigenvalues $E_{i}{ }^{0}=E_{m}{ }^{\mathrm{R}} E_{n}{ }^{\mathrm{T}}$. The total Hamiltonian $H$ for the interacting system $\mathrm{R}+\mathrm{T}$ can be written as $H=H_{0}+U$ where $U$ contains all the interaction terms between $\mathrm{R}$ and $\mathrm{T}$. The Schroedinger equation (S.E.) for the ground state of the interacting system will be denoted by

$$
H \Psi=E \Psi .
$$

In their classic treatment Eisenschitz and London ${ }^{3} \mathrm{ex}-$ pand the wavefunction in terms of antisymmetrized product functions

$$
\Psi=A \Phi_{0}+\sum_{i \neq 0} b_{i} A \Phi_{i} .
$$

The antisymmetrizer $A$ is defined as

$$
A=Q^{-1 / 2} \sum_{P_{\mathrm{RT}}} \delta_{P_{\mathrm{RT}}} P_{\mathrm{RT}},
$$

where $Q$ is the total number of permutations of electrons between the two molecules and the summation over $P_{\mathrm{RT}}$ includes all $Q$ permutations of electrons between $\mathrm{R}$ and $\mathrm{T}$ and $\delta_{P_{\mathrm{RT}}}$ is \pm 1 depending on whether $P_{\mathrm{RT}}$ is an even or odd permutation. The expansion given by Eq. (2) yields the perturbation equations

$$
\begin{gathered}
\left(H_{0}-E_{0}{ }^{0}\right) \Phi_{0}=0 \\
A\left(H_{0}-\mathrm{E}_{0}{ }^{0}\right) \sum_{i} b_{i}{ }^{(1)} \Phi_{i}=A\left(E^{(1)}-U\right) \Phi_{0}, \\
A\left(H_{0}-E_{0}^{0}\right) \sum_{i} b_{i}{ }^{(2)} \Phi_{i} \\
=A\left(E^{(1)}-U\right) \sum_{i} b_{i}{ }^{(1)} \Phi_{i}+A E^{(2)} \Phi_{0},
\end{gathered}
$$


etc., where the $n$ th-order correction to the wavefunction has been written as

$$
\Psi^{(n)}=\sum_{i} b_{i}^{(n)} A \Phi_{i} .
$$

Eischenschitz and London derived solutions for Eqs. (5) and (6) by expanding the right-hand sides of these equations in terms of the functions $\left\{A \phi_{i}\right\}$ and equating the coefficients of these functions on both sides of their equations. Since the functions $\left\{A \phi_{i}\right\}$ are not linearly independent the expansion coefficients for the expansion of an arbitrary antisymmetric function $f$ in terms of these functions are not unique and therefore the results of a perturbation treatment based on such an expansion are not unique.

Following Eisenschitz and London we write the general relation between the functions $\left\{A \Phi_{i}\right\}$ as

$$
A \Phi_{i}=\sum_{j} T_{j i} A \Phi_{j} .
$$

Substituting for $T_{j i}$ the form $T_{j i}=\left\langle\Phi_{j}|T| \Phi_{i}\right\rangle$, one obtains

$$
A \Phi_{i}=A \sum_{j}\left\langle\Phi_{j}|T| \Phi_{i}\right\rangle \Phi_{j}=A T \Phi_{i} .
$$

A general form of $T$ which satisfies Eq. (9) can be written as

$$
T=Q^{-1 / 2} A+T^{\prime},
$$

where $A T^{\prime}=0$. Using this general form of the matrix $T$ in Eq. (8) the consequences of an expansion in terms of the functions $\left\{A \Phi_{i}\right\}$ can be elucidated. Writing $f$ as $f=\sum_{i} d_{i} A \Phi_{i}$ and substituting for $A \Phi_{i}$ according to Eq. (8) yields

$$
f=\sum_{i} \sum_{j} d_{i} T_{j i} A \Phi_{j}=\sum_{j} e_{j} A \Phi_{j}
$$

where

$$
\begin{aligned}
e_{j} & =\sum_{i} d_{i} T_{j i}=\left\langle\Phi_{j} \mid Q^{-1 / 2} \sum_{i} d_{i} A \Phi_{i}\right\rangle+\left\langle\Phi_{j} \mid \sum_{i} d_{i} T^{\prime} \Phi_{i}\right\rangle \\
& =Q^{-1 / 2}\left\langle\Phi_{j} \mid f\right\rangle+\left\langle\Phi_{j} \mid B g\right\rangle .
\end{aligned}
$$

Since the only requirement on $T^{\prime}$ is $A T^{\prime}=0$, the function $\sum_{i} d_{i} T^{\prime} \Phi_{i}$ can be written as $B g$, where $g$ is an arbitrary function depending on the coordinates of $\left(N_{\mathrm{R}}+N_{\mathrm{T}}\right)$ electrons and $B=\left(I-Q^{-1 / 2} A\right)$.

From Eq. (12) it follows that the individual coefficients $e_{j}$ are not uniquely determined by $f$, although the set of coefficients $\left\{e_{j}\right\}$ as a whole uniquely determines $f$. The nonuniqueness of the individual coefficients $e_{j}$ would be without any importance if the results of a perturbation treatment, in which an expansion in terms of the functions $\left\{A \Phi_{i}\right\}$ is used, would be dependent only on the function to be expanded. Although Eisenschitz and London noticed that the results of the separate perturbation equations would be dependent on which expansion coefficients were used, i.e., would be dependent on $g$, they nevertheless restricted their solutions; that is, instead of carrying out a perturbation treatment using the generalized coefficients given by Eq. (12), they selected the expansion which has the property that the sum of the absolute squares of the expansion coefficients is a minimum, i.e., they used the expansion for which $g=0$.

At present many of the difficulties with respect to the perturbation theory for short-range intermolecular forces are related in one way or the other to the nonuniqueness of the expansion of an antisymmetric function in terms of the functions $\left\{A \Phi_{i}\right\}$. It appears to us that a rigorous justification of the choice of Eisenschitz and London is not available, nor indeed is there an adequate justification for any other specific expansion. Therefore a perturbation treatment starting from Eqs. (4)-(6), using the general expansion coefficients (12), has been carried out. The advantages of such a treatment are that it enables one to investigate the explicit dependence of $E^{(1)}, \Psi^{(1)}, E^{(2)}$, etc., on the choice of $g$.

Expansion of the right-hand side of Eq. (5) according to Eq. (12) yields

$$
\begin{aligned}
\sum_{i} b_{i}{ }^{(1)}\left(E_{i}{ }^{0}-E_{0}{ }^{0}\right) A \Phi_{i} \\
\quad=\sum_{i}\left[Q^{-1 / 2}\left\langle\Phi_{i}\left|A\left(E^{(1)}-U\right)\right| \Phi_{0}\right\rangle+\left\langle\Phi_{i} \mid B g_{1}\right\rangle\right] A \Phi_{i},
\end{aligned}
$$

Where the subscript 1 of the function $g$ indicates the $g$ function used in the first-order perturbation equation.

Since the generalized expansion coefficients are used on the right-hand side of Eq. (13), the coefficients of the functions $\left\{A \Phi_{i}\right\}$ may be equated on both sides of that equation without loss of generality. This yields for $i=0$

$$
\begin{array}{r}
E^{(1)}=\left[Q^{-1 / 2}\left\langle\Phi_{0}|A U| \Phi_{0}\right\rangle-\left\langle\Phi_{0} \mid B g_{1}\right\rangle\right] / Q^{-1 / 2} \\
\left\langle\Phi_{0}|A| \Phi_{0}\right\rangle .
\end{array}
$$

For $i \neq 0$ one obtains

$$
b_{i}{ }^{(1)}=\frac{Q^{-1 / 2}\left\langle\Phi_{i}\left|A\left(E^{(1)}-U\right)\right| \Phi_{0}\right\rangle+\left\langle\Phi_{i} \mid B g_{1}\right\rangle}{E_{i}{ }^{0}-E_{0}{ }^{0}} .
$$

If the coefficient $b_{0}{ }^{(1)}$ is taken to be zero, then

$$
\Psi^{(1)}=\sum_{i \neq 0} \frac{Q^{-1 / 2}\left\langle\Phi_{i}\left|A\left(E^{(1)}-U\right)\right| \Phi_{0}\right\rangle+\left\langle\Phi_{i} \mid B g_{1}\right\rangle}{E_{i}{ }^{0}-E_{0}{ }^{0}} A \Phi_{i} .
$$

The second-order perturbation Eq. (6) is resolved in an analogous fashion, i.e., by expanding the right-hand side of Eq. (6) according to Eq. (12) and equating the $\mathrm{Co}^{-}$ efficients of the functions $\left\{A \Phi_{i}\right\}$ on both sides of the equation obtained after expansion. 
For $E^{(2)}$ one obtains

$$
E^{(2)}=-\left[Q^{-1 / 2}\left\langle\Phi_{0} \mid A\left(E^{(1)}-U\right) \sum_{j \neq 0} b_{j}^{(1)} \Phi_{j}\right\rangle+\left\langle\Phi_{0} \mid B g_{2}\right\rangle\right]\left[Q^{-1 / 2}\left\langle\Phi_{0}|A| \Phi_{0}\right\rangle\right]^{-1} .
$$

Substitution of $b_{j}{ }^{(1)}$ according to Eq. (15) into Eq. (17) yields

$$
E^{(2)}=\frac{1}{\left\langle\Phi_{0}|A| \Phi_{0}\right\rangle} \sum_{i \neq 0} \frac{\left\langle\Phi_{0}\left|A\left(E^{(1)}-U\right)\right| \Phi_{i}\right\rangle\left[Q^{-1 / 2}\left\langle\Phi_{i}\left|A\left(E^{(1)}-U\right)\right| \Phi_{0}\right\rangle+\left\langle\Phi_{i} \mid B g_{1}\right\rangle\right]}{E_{0}{ }^{0}-E_{i}{ }^{0}}-\frac{\left\langle\Phi_{0} \mid B g_{2}\right\rangle}{Q^{-1 / 2}\left\langle\Phi_{0}|A| \Phi_{0}\right\rangle} .
$$

The Eqs. (14), (16), and (18) for $E^{(1)}, \Psi^{(1)}$, and $E^{(2)}$, respectively, constitute the results of our generalized treatment.

\section{DISCUSSION OF THE GENERALIZED SOLUTIONS AND RELATION TO OTHER FORMALISMS}

The generalized expressions for $E^{(1)}, \Psi^{(1)}$, and $E^{(2)}$ which show the explicit dependence of these quantities on the functions $g_{1}$ and $g_{2}$ could be very useful in an investigation of the physical meaning of these functions, especially since we have not been able to select a specific $g$ function on mathematical grounds. It is clear that if one carries out a perturbation treatment to infinite order one should use the same $g$ function for each order to obtain unique results. However, if the perturbation treatment is taken to second order only one might ask the question which $g_{1}$ and $g_{2}$ yield the best results for the correction to the energy and the wavefunction.

As has already been mentioned in Sec. II, the Eisenschitz-London treatment corresponds to using an expansion in which $g=0$ and indeed setting $g_{1}=g_{2}=0$ in the generalized expressions for $E^{(1)}, \Psi^{(1)}$, and $E^{(2)}$ yields their well-known results.

Comparing the HAV formalism ${ }^{5}$ and the generalized perturbation treatment presented here it can easily be seen that setting each of the $\phi_{j}$ equal to zero in Eq. (2) of Ref. 5 is equivalent to setting $g=0$. Thus the choice made by Hirschfelder corresponds to selecting a specific expansion of an antisymmetric function in terms of the functions $\left\{A \phi_{i}\right\}$.

Effectively the formalism of Hirschfelder and Silbey ${ }^{6}$ corresponds to choosing ${ }^{7}$

$$
g_{1}=g_{2}=\left(\langle B U\rangle_{0}-U\right) \Phi_{0},
$$

where

$$
\langle B U\rangle_{0}=\left\langle\Phi_{0}|B U| \Phi_{0}\right\rangle /\left\langle\Phi_{0}|B| \Phi_{0}\right\rangle .
$$

From the generalized expression for $E^{(1)}$, Eq. (14), it follows that one can obtain the Heitler-London VB result whenever $\left\langle\Phi_{0} \mid B g_{1}\right\rangle=0$. This requirement is fulfilled in the Eisenschitz-London and HAV formalisms since there $g_{1}=0$, and also fulfilled in the Hirschfelder-
Silbey treatment as can be seen from Eqs. (19) and $(20)$.

In order to make contact with the results for $E^{(1)}$ and $E^{(2)}$ obtained in the Murrell-Shaw ${ }^{8}$ and MusherAmos $^{9}$ formalisms, it is necessary to use functions $g_{1}$ and $g_{2}$ which are not the same. Substituting

$$
\begin{aligned}
& g_{1}=0, \\
& g_{2}=-Q^{-1 / 2} \sum_{i \neq 0} \frac{\left(E^{(1)}-U\right) \Phi_{i}\left\langle\Phi_{i}\left|A\left(E^{(1)}-U\right)\right| \Phi_{0}\right\rangle}{E_{0}{ }^{0}-E_{i}{ }^{0}}
\end{aligned}
$$

into the generalized expressions for $E^{(1)}$ and $E^{(2)}$ yields the same expressions for these quantities as the ones obtained by the aforementioned authors.

\section{SUMMARY}

This generalized treatment shows clearly that the nonuniqueness of the individual expansion coefficients in the representation of an antisymmetric function in terms of the functions $\left\{A \Phi_{i}\right\}$ is the basic reason why so many different results for the first- and second-order equation could be obtained. Using the generalized expressions it can be seen that the difference between the various perturbation formalisms is effectively a result of variations in the choice of the $g$ function.

\section{ACKNOWLEDGMENTS}

One of us (H.N.W.L.) is indebted to Professor D.H.W. den Boer and Dr. F. B. van Duijneveldt, Rijks Universiteit Utrecht, for his initial exposure to theoretical chemistry. The award of a generous Izaak Walton Killam Memorial Predoctoral Scholarship to H.N.W.L. is also acknowledged.

* Predoctoral Killam Fellow.

${ }^{1}$ For an extensive list of literature references see J. O. Hirschfelder, Chem. Phys. Letters 1, 325 (1967).

${ }^{2}$ P. R. Certain et al., J. Chem. Phys. 49, 24 (1968).

${ }^{3}$ R. Eisenschitz and F. London, Z. Physik 60, 491 (1930).

${ }^{4}$ A. Van der Avoird, Chem. Phys. Letters 1, 24 (1967).

${ }^{5}$ J. O. Hirschfelder, Chem. Phys. Letters 1, 363 (1967).

${ }^{6}$ J. O. Hirschfelder and R. Silbey, J. Chem. Phys. 45, 2188 (1966).

${ }^{7}$ Reference 5 contains an improved derivation of the Hirschfelder-Silbey treatment.

${ }^{8}$ J. N. Murrell and G. Shaw, J. Chem. Phys. 46, 1768 (1967). 9 J. I. Musher and A. T. Amos, Phys. Rev, 164, 31 (1967). 